\title{
EFEITOS DA SUPLEMENTAÇÃO DE BEBIDA ENERGÉTICA NA FADIGA MUSCULAR DOS ATLETAS DA EQUIPE MILITAR MASCULINA DE FUTEBOL DO BRASIL
}

\author{
Effects of energy drink supplement with in muscle fatigueof male athletes \\ of brazil military soccer team.
}

\author{
César Augusto Calembo Marra'; Mauro Maia Júnior²; Luciana de Lima e Silva Lauria1 \\ ${ }^{1}$ Instituto de Pesquisa da Capacitação Física do Exército, Rio de Janeiro - RJ \\ ${ }^{2}$ Escola de Educação Física do Exército, Rio de Janeiro - RJ
}

Resumo: INTRODUÇÃO: A suplementação de Taurina e Cafeína individualmente tem demonstrado promover efeitos positivos na performance de equipes desportivas. OBJETIVO: verificar os efeitos da ingestão de suplemento energético a base de Taurina e Cafeína na fadiga muscular dos atletas da equipe masculina de futebol militar do Brasil. METODOLOGIA: Foram selecionados para amostra 20 indivíduos (10 Grupo Teste e 10 Grupo Controle) do sexo masculino, militares, com idade entre 18 e 38 anos, atletas da equipe de futebol das Forças Armadas. A amostra foi dividida aleatoriamente em 2 grupos: Grupo Placebo (GP) $(n=10)$ e Grupo Suplementado (GS) ( $n=10)$. Para a padronização da ingestão da bebida energética uma nutricionista coordenou o consumo desta. Para maior controle, os atletas tiveram que realizar o desjejum (café da manhã) no refeitório do quartel. Logo após o café-da-manhã, o GS ingeriu $300 \mathrm{ml}$ do suplemento energético. O GC recebeu uma bebida placebo. Após a ingestão das bebidas, houve a espera de 90 minutos para absorção do suplemento pelo organismo. Após os 90 minutos, os atletas dirigiram-se ao campo de futebol onde realizaram um prévio aquecimento em torno de dez minutos, seguido de cinco minutos de repouso. Na sequencia do procedimento, foi realizado o teste de potência anaeróbica. O teste consistia na realização de seis corridas de 35 metros em velocidade máxima na grama do campo de futebol e com chuteiras, com intervalo de 10 segundos de recuperação entre as corridas. Após o teste de potencia anaeróbica os atletas participaram de uma partida de futebol com 2 tempos de 45 minutos. Ao término do $1^{\circ}$ tempo da partida, foi realizado um intervalo de 15 minutos e a ingestão de uma nova medida de suplementação e placebo aos atletas, idêntica a suplementação inicial. Após os dois períodos de treino de 45 minutos cada, os jogadores foram novamente submetidos ao teste de potência anaeróbica. Todos os mesmos procedimentos foram executados em duas sessões com um intervalo de 7 dias através de um cruzamento dos grupos (cross-over). Após os 7 dias, os indivíduos do GP passaram a ser GC, bem como os sujeitos do GC passaram a ser GS. RESULTADOS: Os testes de potência anaeróbica antes da incução a fadiga (pré-teste), o tempo médio do GS foi de 4,42 $\pm 0,23$ segundos, já sendo inferior ao tempo dos atletas que consumiram placebo (GP) no qual obtiveram o tempo de 4,44 $\pm 0,27$ segundos. Após o incremento da fadiga (pós-teste) foi observado uma queda na performance em ambos os grupos, considerando que no GC o tempo do teste foi de $4,45 \pm 0,23$ segundos (perda de 0,05 segundos). E no GS o tempo foi de $4,49 \pm 0,22$ segundos (perda de 0,03 segundos). Porém análises estatísticas indicaram que houve diferença significativa entre os grupos, com nível de significância de $p \leq 0,05$. Embora tenha havido uma piora da performance nos testes de potência anaeróbica em ambos os grupos, o grupo que ingeriu a bebida energética (GS) teve uma queda menor da performance do que os sujeitos que ingeriram placebo. Um a variação de 0,04 segundos entre os grupos no pós teste. CONCLUSẢO: Em conclusão, a suplementação de bebida energética contribuiu significativamente para uma menor perda da performance em testes de potência anaeróbica, minimizando a fadiga muscular em atletas com características similares ao do presente estudo.

\begin{abstract}
BACKGROUND: Supplementation of taurine and caffeine alone has been shown to promote positive effects on performance of sports teams. OBJECTIVE: To assess the effects of energy drink supplement with of caffeine and taurine in muscle fatigue of male athletes of Brazil military soccer team. METHODS: We used to sample 20 individuals (10 and 10 Test Group Control Group) were male, men aged between 18 and 38 years, athletes from the soccer team of the Armed Forces. The sample was divided randomly into two groups: Placebo $(G P)(n=10)$ and Drink (GS) $(n=10)$. To standardize the intake of energy drink consumption has coordinated a nutritionist. For more control, the athletes had to make breakfast in the headquarters. Soon after breakfast each morning, the GS ingested $300 \mathrm{ml}$ of supplement. The CG received a placebo drink. After ingestion of the drinks, the wait was 90 minutes for absorption by the body of the supplement. After 90 minutes, the athletes went to the football field where they held a preliminary heating in about ten minutes, followed by five minutes of rest. Following the procedure was performed to test anaerobic power. The test consisted of six sprints of 35 meters at full speed on the grass football field and soccer shoes, with an interval of 10 seconds recovery between sprints. After the test of anaerobic power athletes participated in a soccer game with 2 times of 45 minutes. At the end of the $1 \mathrm{st}$ half of the match, was an interval of 15 minutes and the ingestion of a new measure of placebo and supplementation to athletes, identical to the initial supplementation. After the two practice sessions of 45 minutes each, players were again subjected to the test of anaerobic power. All these procedures were performed in two sessions with an interval of seven days through an intersection of the groups (crossover). After 7 days, individuals in the GP became the GC, as well as the subjects of the group went to GS. RESULTS: Tests of anaerobic power before induction of fatigue (pre-test), the average time of GS was $4.42 \pm 0.23$ seconds, there is less than the time of the athletes who consumed placebo (GP) in which they got time of $4.44 \pm 0.27$ seconds. After the increment of fatigue (post-test) was observed a decrease in performance in both groups, whereas in CG the test time was $4.45 \pm 0.23$ seconds (loss of 0.05 seconds). And the GS time was $4.49 \pm 0.22$ seconds (loss of 0.03 seconds). But statistical analysis indicated a significant difference between groups, with a significance level of $p \leq 0.05$. Although there was a worsening of performance on tests of anaerobic power in both groups, the group that drank the energy drink (GS) had a smaller decline in performance than subjects who took placebo. A variation of 0.04 seconds between the groups on the post test. CONCLUSION: The supplemental energy drink significantly contributed to a minor loss of performance on tests of anaerobic power, minimizing muscle fatigue in athletes with similar characteristics to the present study.
\end{abstract}

Aceito em 14/03/2011 - Revista de Educação Física 2011 Abr; 151:25-31. Rio de Janeiro - Brasil 


\section{INTRODUÇÃO}

O jogo de futebol requer a realização de exercícios físicos intermitentes e prolongados em que se combinam fases curtas de alta intensidade com longos periodos de exercícios de baixa intensida$\mathrm{de}^{(1)}$.

Estudos demonstram a queda no desempenho no segundo tempo de um jogo de futebol que é causada, principalmente, pela fadiga muscular e está associada a alguns fatores tais como, a distância percorrida em alta intensidade ${ }^{(2)}$ e depleção de glicogênio muscular ${ }^{(3,4)}$.

O declínio da força explosiva tem implicações negativas para as ações mecânicas dos jogadores no fim do jogo. Desta forma a fadiga no futebol pode manifestar-se como fator complicador, principalmente, ao final da partida ${ }^{(5)}$.

A suplementação nutricional e o treinamento físico são alguns dos aspectos fundamentais para que o atleta de futebol não tenha queda do desempenho físico durante uma partida ${ }^{(6)}$.

Estudos demonstraram que a suplementação nutricional melhorou o desempenho físico em exercícios de alta intensidade e intermitentes no futebol ${ }^{(7,8)}$.

Bebidas energéticas, tais como, Red Bull Energy Drink $®$ vem sendo investigadas como suplementos para melhorar o desempenho físico ${ }^{(9)}$, produzindo um aumento significativo na resistência muscular em atletas treinados. Essas bebidas energéticas contém várias substâncias que têm múltiplos efeitos no metabolismo humano, por exemplo, a Taurina ${ }^{(10)}$, Glucuronolactona ${ }^{(11)}$, cafeína ${ }^{(12)}$ e vitaminas do complexo $\mathrm{B}^{(13)}$.

Estão bem evidenciadas as ações bioquímicas dos componentes dessas bebidas na melhora do desempenho em atletas treinados ${ }^{(9,14)}$, tornando provável que a ingestão dessa bebida possa ter efeitos importantes na minimização da fadiga muscular em atletas de futebol.

\section{OBJETIVO}

Verificar os efeitos da ingestão de bebida energética na fadiga muscular da equipe militar masculina de Futebol do Brasil.

\section{METODOLOGIA}

\section{Amostra}

Foram selecionados para amostra 20 indivíduos do sexo masculino, militares atletas da equipe de futebol das Forças Armadas em treinamento na Fortaleza de São João (FSJ), sediada no Rio de Janeiro-RJ, Brasil. Os participantes foram informados dos objetivos da pesquisa, dos procedimentos, dos possíveis desconfortos, riscos e benefícios do estudo antes de assinarem o termo de consentimento livre e esclarecido. Os sujeitos que participaram da pesquisa somente foram aceitos como individuos do estudo após uma avaliação médica prévia pelo médico da FSJ. Os indivíduos que tivessem qualquer problema de saúde que impedisse a realização dos exercícios físicos propostos não foram incluídos no estudo.

\section{Local}

O estudo foi realizado na FSJ, quartel do Exército Brasileiro, localizado na cidade do Rio de Janeiro - RJ.

\section{Procedimentos}

Características Físicas da Amostra

Todas as mensurações foram realizadas duas semanas antes dos procedimentos para coleta dos testes de potência anaeróbica. Para isso, no início da manhã no laboratório de Fisiologia do IPCFEx, por volta das 07:30 horas, todos os participantes estavam descalços, com o busto nu e com apenas um calção.

\section{Estatura}

Essa medida consiste na distância entre o vértex e a região plantar, estando a cabeça posicionada com o plano de Frankfurt paralelamente ao solo, e o corpo, na posição anatômica. A aferição foi realizada com o corpo mais alongado possível. Como recomendam Lhoman et al. (1998)(15), as mensurações foram tomadas em triplicata e a média da estatura foi registrada. A estatura foi medida por um estadiômetro (Country Tecnology®, Gays Mills, WI; modelo 67031), com resolução de um centímetro.

\section{Massa Corporal}

A balança utilizada para a mensuração da massa corporal foi a Filizola ${ }^{\circledR}$ eletrônica/digital, 
com resolução de 100 gramas (modelo "Personal Line"). O avaliado posicionou-se em pé, de costas para a escala da balança, com afastamento lateral dos pés, estando a plataforma entre os mesmos. Em seguida, o sujeito colocou-se sobre o centro da plataforma, ereto, com o olhar em um ponto fixo a sua frente.

\section{Consumo Máximo de Oxigênio $\left(\mathrm{VO}_{2 m a ́ x}\right)$}

Com o objetivo de melhor descrever a amostra e auxiliar na prescrição do exercício físico, foi estimado o $\mathrm{VO}_{2 \text { máx }} 2$ semanas antes dos testes de potência anaeróbica.

Para avaliar o $\mathrm{VO}_{2 m a ́ x}$. foi utilizado o teste de Leger \& Lambert (1982)(16) (Shuttle Run test) de 20 metros ida e volta. Os atletas partem de uma marca percorrendo 20 metros em linha reta até a outra marca paralela à outra. A velocidade inicial foi de $8,0 \mathrm{~km} / \mathrm{h}$ com duração de 60 segundos para cada estágio. A velocidade aumentava $0,5 \mathrm{~km} / \mathrm{h}$ por estágio, os atletas foram orientados a completar os estágios dentro de suas possibilidades físicas e o teste foi encerrado quando os atletas abandonarem o estágio ou quando ficarem por duas vezes consecutivas 3 metros atrás da linha dos 20 metros após o sinal do áudio. O protocolo Shuttle Run Test de $20 \mathrm{~m}$ possui equações para predição determinar a velocidade para aqueles que conseguiram completar um dos estágios embora os que não conseguiram completar, tiveram sua velocidade corrigida para ser calculado $\circ \mathrm{VO}_{2 \text { máx. }} \mathrm{O}$ uso deste teste se justifica pelo fato de que no jogo de futebol há constantes mudanças de direção.

\section{Procedimentos dos Testes}

A amostra foi dividida aleatoriamente em 2 grupos: Grupo Placebo (GP) $(n=10)$ e Grupo Suplemetado (GS) $(n=10)$. Para maior fidedignidade dos dados, o estudo foi duplo-cego. Para a padronização da ingestão da bebida energética uma nutricionista coordenou o consumo desta. Nas 24 horas antes dos testes, os sujeitos da amostra foram orientados a não ingerir bebidas que continham produtos que pudessem interferir no estudo, tais como, café preto, chá preto, refrigerantes, chocolates, bebidas energéticas (Ex: Red Bull ou similares) e mates em geral.Para maior controle, os atletas tiveram que realizar o desjejum (café da manhã) no refeitório do quartel.
Logo após o café-da-manhã, o GS ingeriu $300 \mathrm{ml}$ do suplemento energético (Taurina $800 \mathrm{mg} / 200 \mathrm{ml}$, cafeína $64 \mathrm{mg} / 200 \mathrm{ml}$, glucoronolactona 480/200ml). O GC recebeu uma bebida a base de suco e água nas mesmas colorações da bebida energética (suplementação 1). Após a ingestão das bebidas, houve a espera de $90 \mathrm{mi}-$ nutos para absorção do suplemento pelo organismo. Após os 90 minutos, os atletas dirigiram-se ao campo de futebol onde realizaram um prévio aquecimento em torno de dez minutos, seguido de cinco minutos de repouso.

$\mathrm{Na}$ sequencia do procedimento, foi realizado o teste de potência anaeróbica (pré-teste). $O$ teste consistia na realização de seis corridas de 35 metros em velocidade máxima na grama do campo de futebol e com chuteiras, com intervalo de 10 segundos de recuperação entre as corridas.

Após o teste de potencia anaeróbica os atletas participaram de uma partida de futebol com 2 tempos de 45 minutos, em horário das 9 às 11 horas da manhã, em um campo com grama natural e dimensões de $100 \mathrm{~m}$ de comprimento por $70 \mathrm{~m}$ de largura, em intensidade de um jogo profissional normal. Ao término do $1^{\circ}$ tempo da partida, foi realizado um intervalo de 15 minutos e a ingestão de uma nova medida de suplementação e placebo aos atletas, idêntica a suplementação inicial (após o desjejum - suplementação 2). Após o término do $2^{\circ}$ tempo de 45 minutos, os jogadores foram novamente submetidos ao teste de potência anaeróbica (pós-teste).

Todos os mesmos procedimentos foram executados em duas sessões com um intervalo de 7 dias através de um cruzamento dos grupos (crossover). Após os 7 dias, os indivíduos do GP passaram a ser GC, bem como os sujeitos do GC passaram a ser GS.

\section{Análise Estatística}

Para a análise dos dados, foi utilizado o programa Statiscal Package for the Social Sciences (SPSS 12.0 para Windows). A descrição da amostra foi realizada através da estatística descritiva (média e desvio padrão). Todos os dados foram avaliados pela Análise de Variância Split-Plot (SPANOVA). No caso de ocorrerem diferenças significativas na SPANOVA foi utilizado o teste de 
"t" de student (post-hoc) para determinar as diferenças dentro dos grupos (pré-teste e pós-teste).

\section{RESULTADOS E DISCUSSÃO}

As características físicas da amostra podem ser encontradas na Tabela 1. Embora o compromisso extensivo exigido de cada participante, todos os indivíduos completaram todo o estudo ( $n=$ 20) e foram incluídos nas análises finais. A média de idade, altura, peso, Índice de Massa Corporal (IMC) e Consumo Máximo de oxigênio $\left(\mathrm{VO}_{2 m a ́ x}\right)$ não diferiram entre GS e GC.

Tabela 1: Caracteristicas físicas da amostra (média \pm Desvio-padrão)

\begin{tabular}{lcc}
\hline \multicolumn{1}{c}{ Variável } & Grupo Controle & Grupo Suplementado \\
\hline Idade $($ anos $)$ & $26,2 \pm 2,1$ & $27,1 \pm 2,2$ \\
\hline Altura $(\mathrm{cm})$ & $179,2 \pm 4,3$ & $178,3 \pm 3,9$ \\
\hline Peso $(\mathrm{kg})$ & $73,6 \pm 2,6$ & $73,2 \pm 2,7$ \\
\hline $\begin{array}{l}\text { Indice de Massa Corporal } \\
\left(\mathrm{kg} / \mathrm{m}^{2}\right)\end{array}$ & $22,9 \pm 1,9$ & $23,1 \pm 2,4$ \\
\hline $\mathrm{VO}_{2 \text { max }}(\mathrm{ml} / \mathrm{kg} / \mathrm{min})$ & $61,1 \pm 5,4$ & $59,5 \pm 5,1$
\end{tabular}

A utilização dos testes de potência anaeróbica como instrumento de análise observou diferentes gestos motores decisivos em uma partida de futebol que tem como base a potência, explosão e a rapidez dos movimentos em momentos decisivos de um jogo.

Conforme a Tabela 2 pode-se verificar os resultados dos testes de potência anaeróbica antes da indução a fadiga (pré-teste), o tempo médio do GS foi de 4,42 $\pm 0,23$ segundos, sendo inferior ao tempo dos atletas que consumiram placebo (GP) no qual obtiveram o tempo de 4,44 $\pm 0,27$ segundos.

Após o incremento da fadiga (pós-teste) foi observado uma queda na performance, em ambos os grupos, considerando que no GC o tempo do teste foi de 4,49 $\pm 0,23$ segundos (uma piora de 0,05 segundos). E no GS o tempo foi de 4,45 $\pm 0,22$ segundos (uma piora de 0,03 segundos). Porém análises estatísticas indicaram que houve diferença significativa entre os grupos, com nível de significância de $p \leq 0,05$. Embora tenha havido uma piora da performance nos testes de potência anaeróbica em ambos os grupos, o grupo que ingeriu a bebida energética (GS) teve uma redução menor da performance do que os sujeitos que ingeriram placebo. Uma variação de 0,04 segundos entre os grupos no pós teste (Tabela 2).

Dessa forma, a comparação dos suplementados com bebida energética a em relação a ingestão do placebo parece ter exercido um efeito na resistência a fadiga, pois promoveu a redução dos tempos tanto antes quanto após a ingestão das referidas substâncias em relação ao grupo que ingeriu placebo (Figura 1).

Tabela 2. Tempo dos testes de potência anaeróbica pré e pós ingestão de bebida energética em atletas de futebol do Brasil.

\begin{tabular}{cccc}
\hline & $\begin{array}{c}\text { Média } \pm \text { Desvio } \\
\text { Padrão } \\
\text { Pré-teste }(\mathrm{seg})\end{array}$ & $\begin{array}{c}\text { Média } \pm \text { Desvio - } \\
\text { Padrão } \\
\text { Pós-teste }(\mathrm{seg})\end{array}$ & $\Delta$ (seg) \\
\hline $\begin{array}{c}\text { Grupo } \\
\text { Controle }\end{array}$ & $4,44 \pm 0,27$ & $4,49 \pm 0,22$ & $-0,05$ \\
\hline Grupo & $4,42 \pm 0,23$ & $4,45 \pm 0,23$ & $-0,03\left(^{*}\right)$
\end{tabular}

Sulpementado

Nota: onde ${ }^{*} p<0,05$ entre o pré e o pós-teste, $\Delta=$ tempo inicial - tempo final

Essa diferença na performance entre os grupos parece ter explicações nos efeitos das substancias presentes nas bebidas energéticas no metabolismo humano, como, Taurina10, Glucuronolactona11, Cafeína12 e vitaminas do complexo B13.

As várias ações bioquímicas da Taurina14, juntamente com a constatação de que a Taurina e a Glucuronolactona terem um efeito combinado na endurance em atletas treinados9, tornam provável que a Taurina tenha efeitos positivos sobre a resistência muscular.

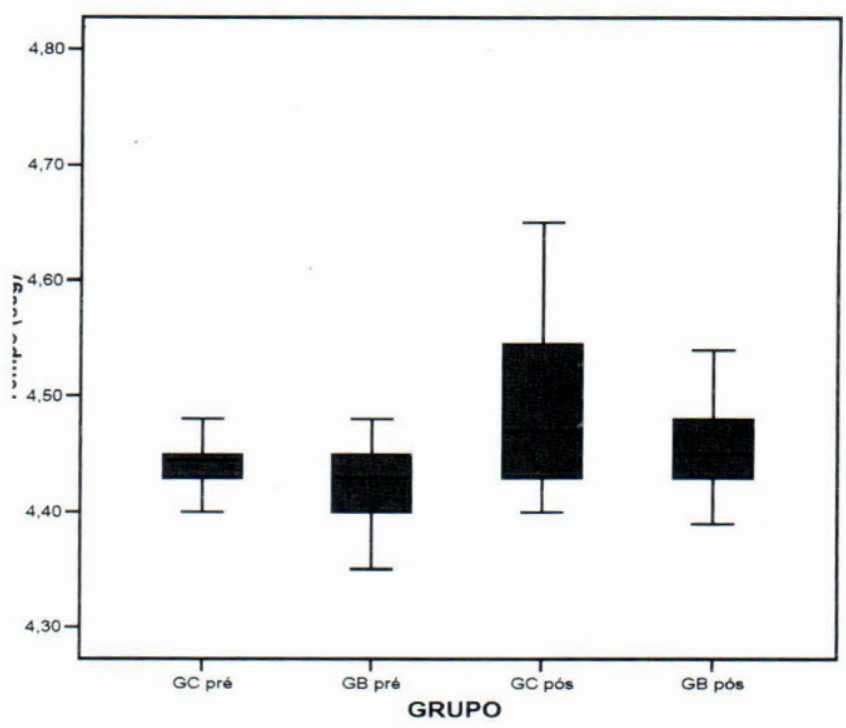

Figura 1: Alterações nos tempos dos grupos controle e bebida nos pré e pós teste. 
Alford et al. (2001)(17) comprovaram consistentemente os efeitos positivos da ingestão de bebidas energéticas no desempenho muscular mostrando que resultados positivos foram encontrados, incluindo tarefas psicomotoras e cognitivas.

Efeitos fisiológicos da taurina contida em uma bebida energética foram observados em indivíduos fisicamente ativos ${ }^{(18)}$. Um aumento de 10 watts com a administração da bebida energética, contudo, sem significância estatística, indicando que a administração de $2 \mathrm{~g}$ de taurina contida em bebidas energéticas não foi capaz de melhorar o desempenho.

Alguns estudos têm mostrado que a cafeína pode aumentar o desempenho físico, a capacidade de resistência, incluindo a força muscular e a de pico ${ }^{(19,20)}$. O bloqueio do receptor de adenosina é uma ação do princípio da cafeína resultando em um efeito estimulante(21).

O que parece explicar a ação da cafeína e taurina no aumento da potência muscular e conseqüente acréscimo da resistência a fadiga é o aumento da velocidade sináptica de impulsos nervosos $^{(22)}$; a maior disponibilidade de cálcio para a potencialização da contração muscular ${ }^{(23)}$; e a concorrência com os receptores de adenosina no sistema nervoso central que age impedindo a ação do sintoma de fadiga durante a realização de exercícios físicos ${ }^{(24)}$.

Schneiker et al. (2006) ${ }^{(25)}$ submeteram atletas de diferentes esportes coletivos, inclusive jogadores de futebol, à exe $\neg$ cução de sprints intermitentes em ciclo ergométrico, e observou aumento significativo na quantidade de trabalho executado e no pico de potência atingido por esses atletas com a ingestão de $6 \mathrm{mg} / \mathrm{kg}$ de cafeína por peso corporal.

Essas bebidas energéticas também contém vitaminas do grupo $B$.

A vitamina B12 pode facilitar o desempenho mental, incluindo a concentração(26), e a recuperação do estado de fadiga muscular e aumento do desempenho físico através da maior produção de energia(13).

Além desses compostos, as bebidas energéticas também contém glicose, um importante substrato metabólico. A glicose é metabolizada para liberação de energia durante a aeróbica e anaeróbica metabolismo ${ }^{(27)}$ e parece melhorar o desempenho cognitivo onde parece contribuir para melhor desempenho físico. Sobre o desempenho muscular, observou que estoques reduzidos do conteúdo de glicogênio reduziram tanto a capacidade aeróbia como a capacidade de realizar atividades de alta intensidade ${ }^{(28)}$.

O autor verificou que jogadores de futebol com conteúdo baixo de glicogênio na musculatura dos membros inferiores (quadríceps) logo no início do jogo percorreram uma distância $25 \%$ menor do que os outros jogadores. No entanto, as diferenças mais marcantes foram notadas nas atividades realizadas em alta velocidade, ie, jogadores com estoques de glicogênio inicialmente baixos percorreram $50 \%$ do total da distância caminhando e $15 \%$ em velocidade, ao passo que jogadores com altos níveis de glicogênio muscular logo no início da atividade percorreram $27 \%$ caminhando e $24 \%$ participando de corridas executadas com velocida$\mathrm{de}^{(29)}$.

\section{CONCLUSÃO}

Em conclusão, no presente estudo, ambos os grupos suplementados e placébo tiveram queda na performance após uma partida de futebol. Porém, a suplementação de bebida energética contribui significativamente para uma menor perda da performance em testes de potência anaeróbica, minimizando a fadiga muscular.

Portanto, parece que a ingestão de bebida energética parece ter efeitos ergogênicos em jogadores de futebol, dando uma maior resistência à fadiga ao apresentar uma menor perda da performance em testes de potência anaeróbica em atletas com características similares ao do presente estudo.

\section{REFERÊNCIAS BIBLIOGRÁFICAS}

1- Rebelo A, Krustrup P, Soares J, Bangsbo J. Reduction in intense intermittent exercise performance during a soccer match. Proceedings of the Second Annual Congress of the European College of Sport Science Sport Science in a Changing World of Sports, 1997. 
2- Bangsbo J, NOrregaard L, ThorsOe F. Activity profile of competition soccer. Can J Sport Sci 1991; 16: 110-116.

3- Gollnick, P.D. Peripheral factors as limitations to exercise capacity. Canadian Journal of Applied Sport Sciences 1982; 7, 14-21.

4- Sahlin K, Katz A, Broberg S. Tricarboxylic acid cycle intermediates in human muscle during prolonged exercise. American Journal of Physiology 1990; 259:834-841.

5- Rahnama N, Reilly $T$, Lees A. Does muscle performance changes during a soccer game. Cellular \& Molecular Biology Letters 2000; 9: 113-116.

6- Guerra I, Soares EA, Burini RC. Aspectos nutricionais do futebol de competição. Rev Bras Med Esporte 2001; 7 (6): 200-206.

7- Maughan RJ, Merson NP. Broad and S.M. Shirreffs. Fluid and electrolyte intake and loss in elite soccer players during training. Int. J. Sport Nutr. Exerc. Metab 2004; 14:333-346.

8- Guerra I, Chaves R, Barros T, Tirapegui J. The influence of fluid ingestion on performance of soccer players during a match. Journal of Sports Science and Medicine 2004; 3: 198-202.

9- Geiss K-R, Jester I, Falke W, Hamm M, Waag $\mathrm{KL}$. The effect of a taurine containing drink on performance in 10 endurance-athletes. Amino Acids $1994 ; 7: 45-56$

10- Stapleton PP, O'Flaherty L, Redmond HP, Bouchier-Hayes DJ. Host defense - a role for the amino acid taurine? J Parenter Enteral Nutr 1998; 22/1: 42-48.

11- Poon R, Villeneuve DC, Chu I, Kinach R. HPLC determination of D-glucaric acid in human urine. $\mathrm{J}$ Anal Toxicol 1993; 17: 146-150.

12- Arnaud MJ. Pharmacokinetics and metabolism of caffeine. In: Snel J, Lorist MM (eds) Nicotine, caffeine and social drinking: behaviour and brain function. Harwood Academic Publishers, 1998, Amsterdam, p 153

13- McArdle WD, Katch FI, Katch VL. Essentials of exercise physiology. Lea and Febiger, Philadelphia, 1994.
14- Huxtable RJ. Physiological actions of taurine. Physiol Rev 1992; 72/1: 101-163.

15- Lohman TG, Roche A, Martorell R. Anthropometric standardization reference manual. Champaign, IL. Human Kinetics, 1998.

16- Leger LA, Lambert J. A maximal multistage 20-m shuttle run test to predict VO2 máx. European Journal Of Applied Physiology And Occupational Physiology 1982; 49: 1 -12.

17- Alford $\mathrm{CH}$, Wescott R. The effects of Red Bull Energy Drink on human performance and mood. Amino Acids 2001; 21: 139-150.

18- Dall'Agnol T, Souza PFA. Efeitos Fisiológicos Agudos da Taurina Contida em uma Bebida Energética em Individuos Fisicamente Ativos Rev Bras Med Esporte 2009; 15 (2): 123-126.

19- Anselme F, Collomp K, Mercier B, Ahmaidi S, Prefaut $C$. Caffeine increases maximal anaerobic power and blood lactate concentration. Eur J Appl Physiol 1992; 65: 188-191.

20- Meliska CJ, Lawson CF. Effects of caffeine and sugar-free beverages on psychomotor performance. Perc Mot Skills 1996; 82: 912-914.

21- Fredholm BB. Adenosine, adenosine receptors, and the action of caffeine (Astra Award Lecture). Pharmacol Toxicol 1995: 76: 93-101.

22- Graham TE, Spriet LL. Performance and metabolic responses to a high caffeine dose during prolonged exercise. J Appl Physiol 1991; 21: 2292-2298.

23- Tarnopolsky M, Cupido C. Caffeine potentiates low frequency skeletal muscle force in habitual and no habitual caffeine consumers. J Appl Physiol 2002;89:1719-24.

24- Laurent D, Schneider KE, Prusaczyk WK, Franklin C, Suzanne VM, Petersen KF, et al. Effects of caffeine on muscle glycogen utilization and the neuroendocrine axisduring exercise. J Clin Endocrinol Metab 2000; 85:2170-5. 
25- Schneiker KT, Bishop D, Dawson B, Hackett LP. Effects of caffeine on prolonged intermittent-sprint ability in team-sport athletes. Med Sci Sports Exerc. 2006; 38:578-85.

26- Mayer G, Kroger M, Meier-Ewert K. Effects of vitamin B12 on performance and circadian rhythm in normal subjects. Neuropsychopharmacol 1996; 15/5: 456-464.

27- Sizer FS, Whitney EN. Nutrition: concepts and controversies. Wadsworth Publishing Company, Belmont, 1997.
28- Ekblom B. Applied physiology of soccer. Sports Medicine 1986; 3: 50-60.

29- Filho HT. Modelos de treinamentos híbridos e desempenho esportivo. Revista Digital - efesportes 1999; 14, 139.

Endereço para correspondência:

César Augusto Calembo Marra

Avenida João Luis Alves, s $/ n^{\circ}$ Fortaleza de São João Urca - Rio de Janeiro - RJ

Email: cesarmarra@gmail.com 